\title{
Caracterización florística de un paisaje antrópico con árboles remanentes en el Valle Central Occidental de Costa Rica
}

\author{
Verónica Bonilla Villalobos \\ Universidad Estatal a Distancia, Costa Rica. Maestría en Manejo de Recursos Naturales. Escuela de \\ Ciencias Exactas y Naturales. Correo: veronicabonillav@gmail.com
}

Recibido: 03 de Junio de 2019

Aceptado: 10 de marzo de 2020

\section{RESUMEN}

Con la finalidad de describir la composición florística de la finca Los Llanos, (renombrada por la UNED, Estación Experimental Los Llanos, según acuerdo Rectoría CR 2019-914), se realizó una caracterización florística del bosque húmedo premontano transición a basal en un paisaje degradado ubicado en la comunidad de Los Llanos, Alajuela. El trabajo se realizó entre 2016 y 2017, durante de siete meses. Se establecieron aleatoriamente 20 parcelas circulares donde se contabilizaron e identificaron todas las especies de plantas con un diámetro (DAP) mayor o igual a 10 $\mathrm{cm}$. Se obtuvo información sobre: a) el estado actual del ecosistema, b) muestreo rápido de especies forestales, c) identidad taxonómica de las especies, d) gremios ecológicos de las especies, e) área basal, f) análisis de diversidad alfa y g) caracterización del suelo y h) recurso hídrico. El sitio es atravesado por la quebrada Ojo de Agua y cuenta con un bosque ribereño alterado, que colinda con dos nacientes de agua registradas. Abundan las plantas herbáceas, mayoritariamente de las familias Asteraceae, Poaceae, Verbenaceae, así como de cultivos mixtos como Phaseolus vulgaris y Zea mays. El sitio tiene suelos muy pedregosos y arcillosos (vertisoles), una cobertura forestal dispersa, con presencia de algunos árboles con alto valor comercial especialmente cedro (Cedrela odorata), cenízaro (Samanea saman) y guanacaste (Enterolobium cyclocarpum) y el bosque ribereño está compuesto principalmente de especies como roble sabana (Tabebuia rosea), yos (Sapium glandulosum), cedro (Cedrela odorata) y guarumo (Cecropia obtusifolia). Además, la cobertura forestal riparia es discontinua. Este estudio encontró una composición florística del bosque transicional muy alterada, pero con algunas especies representativas del Valle Central que pueden colaborar como remanentes para iniciar un proceso de restauración ecológica en la comunidad de Los Llanos.

Palabras claves: Restauración ecosistemas, reforestación, especies nativas, bosque húmedo premontano, caracterización florística.

\author{
ABSTRACT: Floristic characterization of an \\ anthropic landscape with remnant trees in the \\ Central Western Valley of Costa Rica.
}

In order to describe the floristic composition of Los Llanos estate, a floristic characterization of the premontane humid forest transition to basal was performed in a degraded landscape located in the community of Los Llanos, Alajuela. The work was carried out between 2016 and 2017, for an approximately seven months. A total of twenty circular plots were randomly established, where all plant species with a diameter at breast height (DBH) greater than or equal to $10 \mathrm{~cm}$ were counted and identified. Information was obtained on: a) the current state of the ecosystem, b) rapid sampling of forest species, c) taxonomic identity of the species, d) ecological guilds of the species, e) basal area, f) analysis of alpha diversity g) soil characterization and $h$ ) water resource status. The site is crossed by the Ojo de Agua creek, which has an altered riparian forest and borders two registered springs of water. Much of the territory is dominated by herbaceous plants, mostly from the Asteraceae, Poaceae and Verbenaceae families, as well as mixed crops such as Phaseolus vulgaris and Zea mays. The site is characterized by very stony and clayey soils (vertisols), a dispersed forest cover with the presence of some trees with high commercial value, especially Cedrela odorata, Samanea saman and Enterolobium cyclocarpum and the riparian forest is mainly composed of species such as Tabebuia rosea, Sapium glandulosum, Cedrela odorata and Cecropia obtusifolia. In addition, riparian forest cover is discontinuous as well as the crop areas within the farm. This study found a highly altered transitional forest floristic composition, with some representative species from the Central Valley that can collaborate as remnants to initiate an ecological restoration process in the community of Los Llanos.

Key words: Restoration ecosystems, reforestation, native species, premontane wet forest, floristic characterization. 


\section{Introducción}

La caracterización de la vegetación es fundamental para conocer la dinámica de los diferentes aspectos ecológicos que presenta un sitio (Bawa y McDade, 1994). De una adecuada caracterización vegetal se obtienen datos para la implementación de medidas adecuadas para la conservación y manejo de un área a largo plazo, especialmente áreas reducidas o fragmentadas (Cascante y Estrada, 2012).

El tema de restauración ecológica es relativamente de reciente aplicación en Costa Rica y, a pesar de la existencia de algunas experiencias en otras partes del territorio nacional, en la zona de vida bosque húmedo premontano con transición a seco (bosques tropicales estacionalmente secos), son muy pocas las investigaciones realizadas. La vegetación original que corresponde a este ecosistema ha desaparecido casi por completo y no existen áreas de tamaño significativo de bosque primario (Cascante y Estrada, 2012). Se estima que tan sólo un $21 \%$ del bosque original se mantiene aún hoy día, presentando un alto grado de fragmentación y conformado por pequeños remanentes o islas de bosques relativamente aisladas (Sánchez y Azofeifa, 2001).

En esta zona de vida se ha concentrado la mayor parte de la población del país y una de las principales razones es porque los suelos son fértiles por su origen volcánico y proporcionan un desarrollo dinámico de diversas actividades agropecuarias (Fournier, 1985).

La cobertura vegetal actual de la finca Los Llanos está compuesta, por una matriz de pastizales exóticos con la presencia dispersa de vegetación arbórea nativa. La degradación del suelo por el uso extensivo de la ganadería y los monocultivos ocasionaron la pérdida de esta cobertura, constituyendo amenazas contra su integridad, aunado a la escasa información básica para revertir ese proceso (Bonilla, 2018).

Asimismo, estos agropaisajes por lo general son dominados por una matriz de potreros o cultivos anuales, pero aún retienen alguna cobertura arbórea dispersa en forma de pequeños parches remanentes de bosques, franjas angostas de bosques ribereños y árboles dispersos (Sánchez, Harvey, Grijalva, Medina, Vílchez \& Hernández, 2005). Harvey et al., (2004) indican que en algunas ocasiones estos paisajes incluyen cercas vivas para separar repastos o bien como límite entre propiedades, tal como ocurre en el caso de la finca Los Llanos especialmente en lo que se refiere a límite del predio.
Con el fin de documentar la diversidad florística en una de las zonas de vida más alteradas especialmente en el centro del país, se llevó acabo esta investigación en una finca ubicada en La Garita de Alajuela, cuyo uso intensivo y extensivo de la ganadería y cultivos agrícolas provocó un proceso de degradación de la vegetación natural.

\section{Metodología}

La investigación se llevó a cabo en la Finca Los Llanos entre los años 2016 y 2017, durante aproximadamente siete meses (setiembre a marzo) y presenta una extensión de 9.6 hectáreas (ha).

Área de Estudio: La investigación se desarrolló en la finca Los Llanos, la cual presenta una cobertura boscosa muy degrada (Alajuela, La Garita, 9॰58'44.94” N, $84^{\circ} 18^{\prime} 7.056^{\prime \prime}$ W, a los 500 m.n.s.m), está compuesta por pastizales y alguna vegetación arbórea nativa muy dispersa, por lo que se puede considerar un sitio degradado. Esta finca colinda al norte, este y oeste con una propiedad de la Hacienda Siquiares Ltda y la Asociación de la Vivienda de La Garita; al sur con la calle pública de Llanos y con la propiedad de Rafael Sandoval Brenes (IMAS, 2013) (Figura. 1). Colinda con dos nacientes de agua registrada, una quebrada permanente y otra intermitente reconocidas en los mapas oficinales del Instituto Geográfico Nacional. La cobertura vegetal actual de la finca se compone por pastizales de origen exótico y alguna vegetación arbórea nativa dispersa. Se ha generado una pérdida biodiversidad, debió al cambio de uso de suelo, como el uso extensivo de la ganadería, los monocultivos y la pérdida casi total de la cobertura boscosa, estas acciones aceleran procesos que el suelo no está en condiciones de soportar conduciendo a su degradación (Figura. 1).

Análisis y recolección de datos. Se establecieron aleatoriamente 20 parcelas circulares de $500 \mathrm{~m}^{2}$, (radio $12,56 \mathrm{~m}$ ), se contabilizó e identificó todas las especies de plantas con un diámetro (DAP) mayor o igual a $10 \mathrm{~cm}$ (Figura 2). Las parcelas fueron seleccionadas mediante la herramienta Hawths Tools v3.27 (2006) dentro del programa ArcGis v10.3 y por medio de los módulos Create Vector Grid y Create Random Selection, se crearon cuadrículas correspondientes a las parcelas y se seleccionaron al azar las unidades de muestreo. Una vez seleccionadas las celdas al azar se utilizó la herramienta ETGeowizards v11.0 con el módulo Polygon to Point, con el fin de tener la coordenada central de cada celda. 

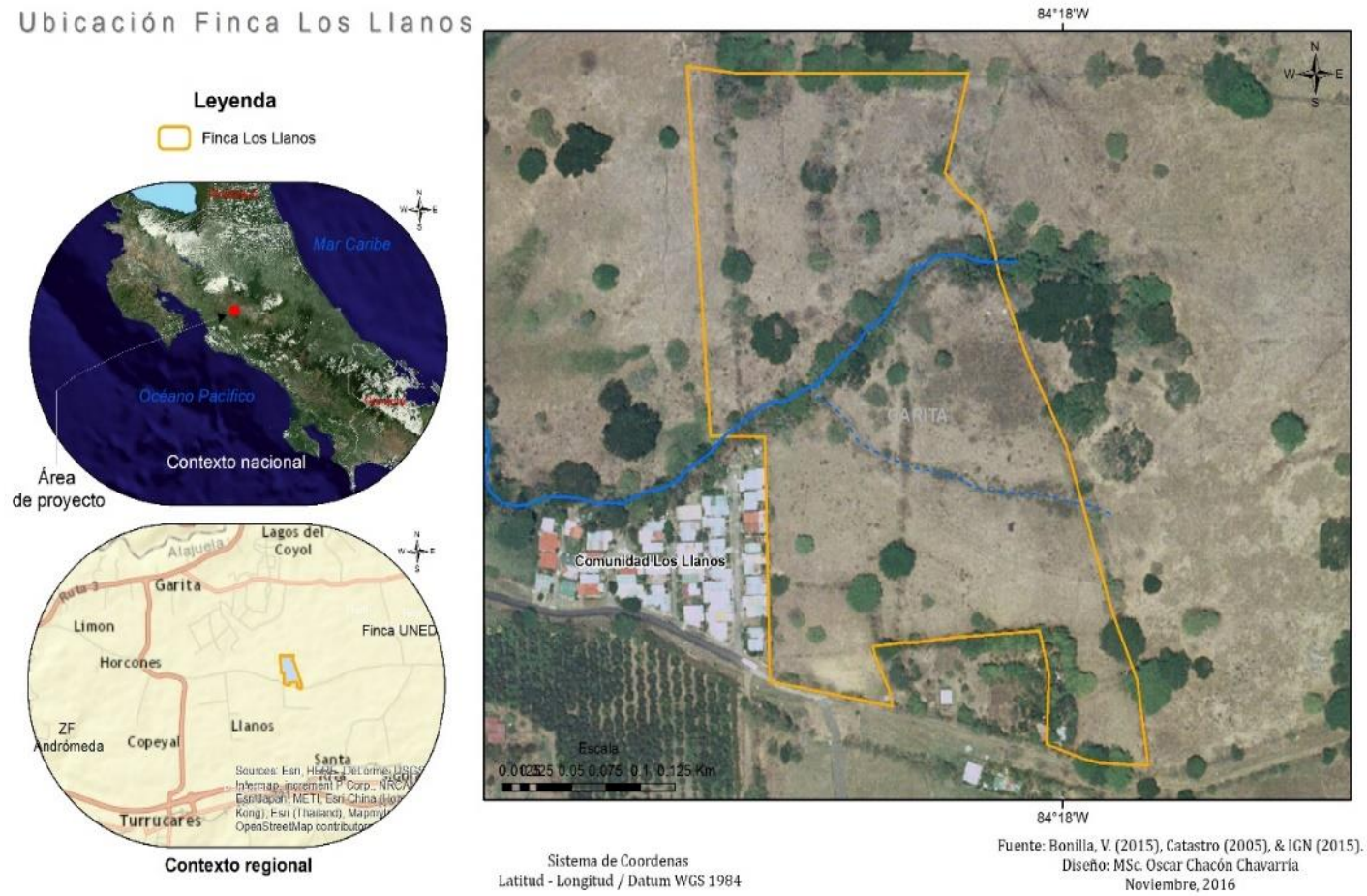

Figura. 1. Ubicación del área de estudio; finca Los Llanos, La Garita, Alajuela, Costa Rica.

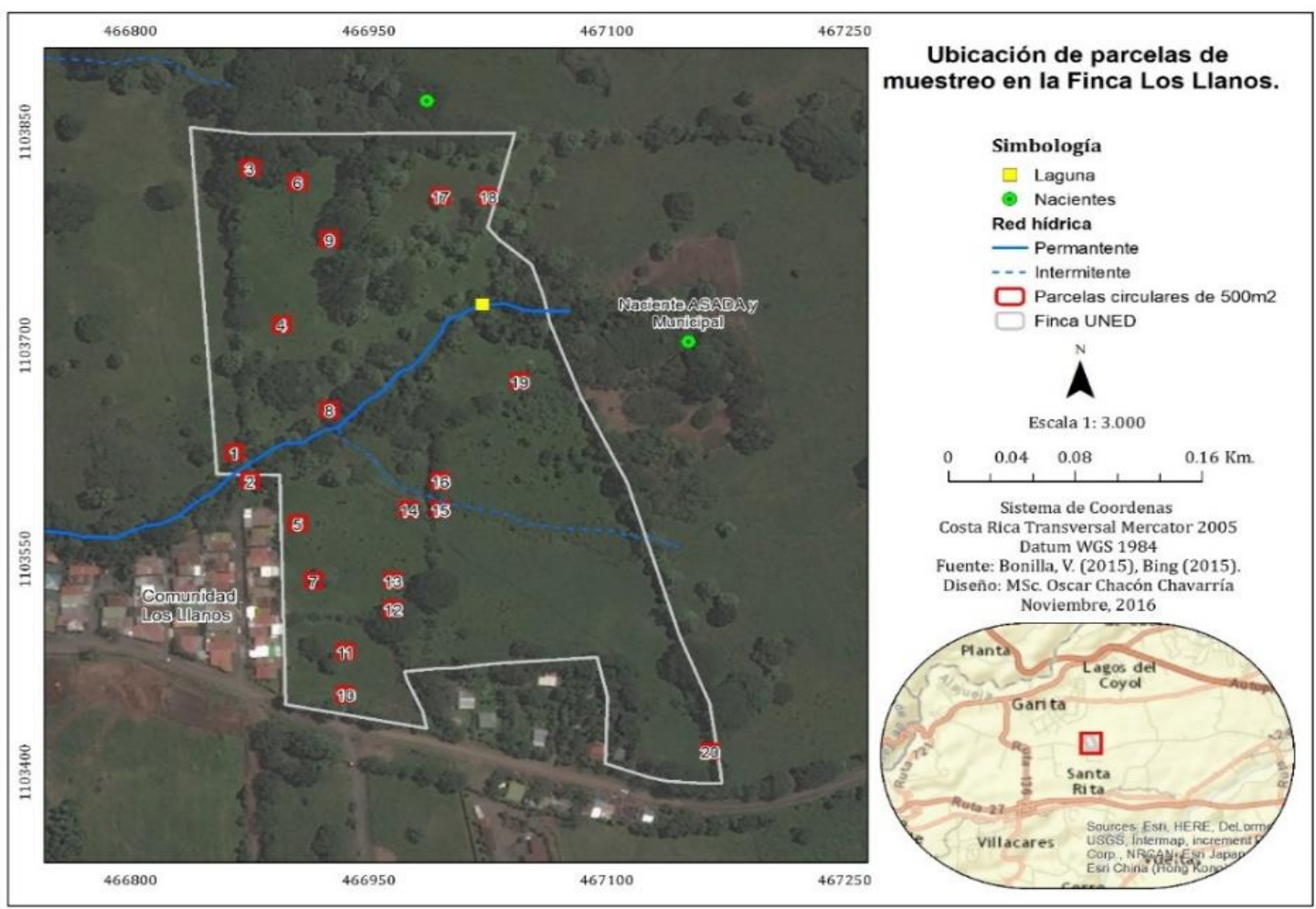

Figura 2. Ubicación de las unidades de muestreo en la finca Los Llanos, La Garita, Alajuela. 
También, se realizó una caracterización del sitio de estudio donde se trabajó en ocho criterios que fueron seleccionados durante de las visitas al campo y los muestreos realizados: a) descripción del estado actual del ecosistema, b) muestreo rápido especies forestales, c) identidad taxonómica de las especies, d) clasificación de las especies en sus gremios ecológicos, e) área basal de las especies, f) análisis de diversidad alfa (estimaciones de abundancia, frecuencia y dominancia, IVI, el índice de valor de importancia (IVI) y el índice de importancia familiar o FIV, g) caracterización física del suelo y h) recurso hídrico. (Mori, Boom, Carvalho, y Dos Santos, 1983; Lamprecht,1990; Moreno 2001; Mostacedo y Fredericksen, 2000; Villarreal 2006).

Para las primeras cuatro actividades se utilizó el método de observación directa, se contó con la ayuda de un especialista para la identificación de las especies forestales, se apoyó con guías taxonómicas de flora y la base de datos de la Comisión Nacional para el Conocimiento y Uso de la Biodiversidad (CONABIO), además, se utilizó la base de datos del sistema de información de recursos naturales para la clasificación de las especies según su gremio ecológico. Con respecto a las otras cuatro se utilizó las fórmulas respectivas para cada uno de los índices.

\section{Resultados}

Existe presencia de vegetación riparia alterada y en la intermitente situada más hacia el extremo sur de la propiedad no hay presencia de vegetación arbórea debido al histórico uso de pastizales en esta sección de la propiedad. A raíz de lo anterior, según lo establecido por ley forestal $\mathrm{N}^{\circ} 7575$ y ley de aguas $\mathrm{N}^{\circ} 276$, un $64.52 \%$ (6.138ha) de la propiedad debe ser protegida en dos áreas, una de protección o amortiguamiento de las nacientes de $61.27 \%$ (5,82ha) y de la quebrada de $3.34 \%$ (0,318ha) y un 35,39\%(3.362ha) de la finca está disponible para cualquier otro uso (Figura 3).

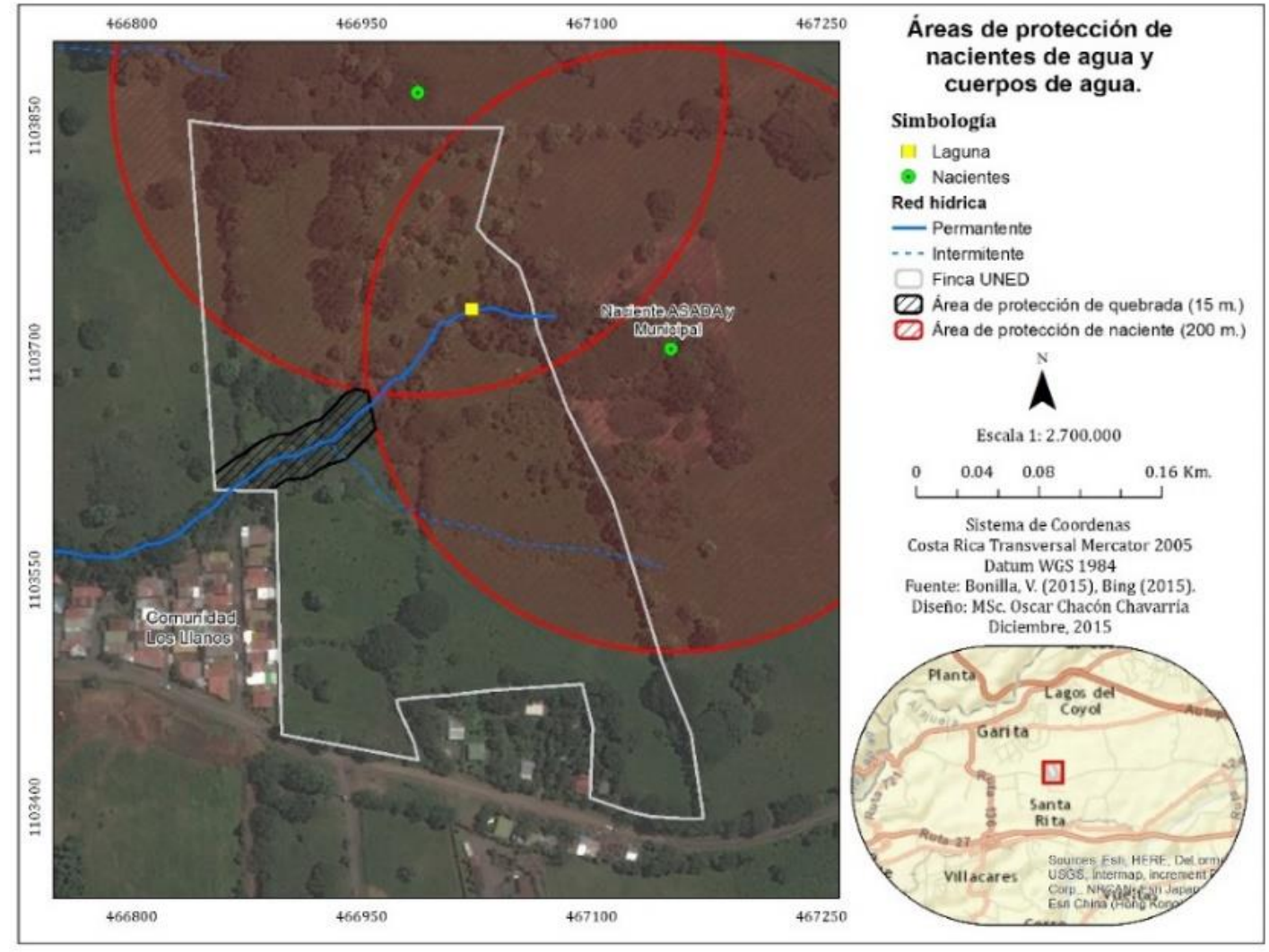

Figura. 3. Zonificación del área de protección dentro de la finca Los Llanos, La Garita, Alajuela. 
La composición de plantas herbáceas se distingue por presentar especies diferentes en dos sectores específicos, el primero se encuentra fuera del área anegada y está dominado por especies de las familias Asteraceae, Poaceae, Verbenaceae, además, Lantana camara, Ipomoea sp, así como de cultivos mixtos Phaseolus vulgaris y Zea mays. Otra sección de este primer sector presenta especies como Vernonia patens, Hyptis suaveolens, Mimosa pudica, Sida acuta. Finalmente, una tercera sección del sector uno presenta un proceso de regeneración masiva de Vernonia patens, Malpighia glabra y de Enterolobium cyclocarpum y de plántulas de Sapium glandulosum. El sector dos corresponde con el sitio cercano a la quebrada donde se presenta la dominancia Solanum seaforthianum y Malpighia glabra siendo enredaderas con densas poblaciones. En otras partes de la finca donde el suelo presenta condiciones de anegamiento es posible identificar pastos de la familia Cyperaceae, así como algunos individuos de Mimosa pigra, entre otros.

La finca se caracteriza por presentar: a) suelo muy pedregoso y arcilloso (vertisol), b) cobertura forestal intervenida, con presencia de árboles con alto valor comercial, c) bosque ribereño fragmentado, sin continuidad y compuesto, principalmente, de especies como roble sabana (Tabebuia rosea), yos yos (Sapium glandulosum), cedro (Cedrela odorata), guarumos (Cecropia obtusifolia), con sotobosque en sus alrededores; d) áreas de cultivos dentro de la finca; e) presencia de dos áreas de protección de nacientes las cuales se sitúan en las cercanías de la propiedad, en dirección Norte y Este respectivamente.

Por otro lado, según la información de la UNED, se encontró una primera propuesta de un Plan Maestro para la construcción de infraestructura el cual parece no haber considerado el radio de protección de una de las nacientes según lo establecido en la legislación nacional con respecto a la localización del salón de multiusos.

El proceso de identificación de las especies arbóreas se llevó a cabo en dos momentos y con dos procesos distintos. Durante el primero se implementó un muestreo rápido de observación caracterizado por recorridos a lo largo de la finca, lo cual permitió obtener una visión global de las especies presentes, obteniendo un total de 39 especies correspondientes a 21 familias (Cuadro 1).
Posteriormente se establecieron 20 parcelas de muestreo al azar, identificando una riqueza de 18 especies, correspondientes a 16 familias, donde la Fabaceae Mimosoidea y la Burseraceae tiene la mayor cantidad de individuos, mientras que cinco de ellas están representada con al menos cinco individuos (Cuadro 2).

Las especies vegetales dependen de su respuesta a la presencia de "claros" (áreas despejadas) y la correspondiente exposición a la luz, desde esta perspectiva, la mayoría de las especies identificadas en la propiedad se refieren a especies del gremio ecológico heliófitas durable. Observando la ausencia de estratos, se encontró presencia de árboles dispersos y de especies de alto valor comercial como el espavel (Anacardium excelsum), guanacaste (Enterolobium cyclocarpum), cenízaro (Samanea saman), cedro (Cedrela odorata), que en su mayoría son de gran altura y de gran fuste (Cuadro 3).

En cuanto al área basal (indicador más heterogéneo del índice de ocupación) de las parcelas, tres de ellas no presentaron cobertura vegetal arbóreas (P4, P6, P14), la parcela P18 mostró mayor área basal con $22,18 \mathrm{~m}^{2}$ ha. Esto evidencia la degradación presente en el sitio de estudio, asimismo, se obtuvo que el área basal promedio por hectárea fue de $76,60 \mathrm{~m}^{2} / \mathrm{ha}(\mathrm{min} .=0,007$; máx.= 14,52; D.E.= 2,22) (Figura 4).

Se identificaron las 10 primeras especies con mayor área basal, de las cuales resalta la especie Enterolobium cyclocarpum $\left(18.62 \mathrm{~m}^{2} / \mathrm{ha}\right)$ seguida por Ficus $s p$ $\left(14.71 \mathrm{~m}^{2} / \mathrm{ha}\right)$ y en un tercer lugar Guazuma ulmifolia $y$ Tabebuia rosea (10.41m2/ha) (Figura 5).

Por otra parte, se calculó el área basal de las familias de flora muestreadas y se enumeran las 10 primeras familias, donde la Fabaceae Mimosoidea es la que presenta una mayor área basal, seguida de la Moraceae. Todas las especies como familias presentes son consideradas representativas de la zona de vida bh-P transición a basal (Figura 6).

En cuanto a la distribución diamétrica, se identificaron 34 individuos (45\%) con un $\mathrm{DAP} \geq 80 \mathrm{~cm}$, lo cual podría estar asociado a la escasa competencia por nutrientes, agua y espacio, además, se identificó que un $9 \%$ de los individuos se encuentran entre $30-34,99 \mathrm{~cm}$; un $10 \%$ con un DAP entre $15-19,99 \mathrm{~cm}$, el restante se distribuye entre porcentajes menores (Cuadro 4). 
Cuadro 1. Muestreo rápido de especies de flora registradas en la finca Los Llanos, 2016 - 2017.

\begin{tabular}{|c|c|c|}
\hline Familia & Nombre Científico & Nombre Común \\
\hline Anacardiaceae & $\begin{array}{l}\text { Anacardium excelsum (Bertero \& Balb. Ex } \\
\text { Kunth) Skeels }\end{array}$ & Espavel \\
\hline Anacardiaceae & Spondias purpurea, $($ L.) & Jocote \\
\hline Anacardiaceae & Tapirira guianensis, (Aubl.) & Manteco \\
\hline Bignoniaceae & Tabebuia rosea, (Bertol.) DC. & Roble de sabana \\
\hline Burseraceae & Bursera simaruba, (L.) Sarg. & Indio desnudo \\
\hline Euphorbiaceae & Sapium glandulosum, (L.) Morong & Yós yós \\
\hline Fabaceae Caesalpinioidea & Cassia fistula, Benth. & Caña fistula \\
\hline Fabaceae Mimosoidea & Enterolobium cyclocarpum, (Griseb.) & Guanacaste \\
\hline Fabaceae Mimosoidea & Inga densiflora, (Benth) & Guaba \\
\hline Fabaceae Mimosoidea & Leucaena leucocephala, (Lam.) de Wit & Guaje, guaje blanco, guaje verde \\
\hline Fabaceae Mimosoidea & Samanea saman, (Jacq.) Merr. & Cenízaro \\
\hline Fabaceae Mimosoidea & Zygia longifolia, (Britton) & Sotacaballo \\
\hline Fabaceae Papilionoidea & Diphysa americana, (Sousa $S$ ) & Guachipelín \\
\hline Fabaceae Papilionoidea & $\begin{array}{l}\text { Gliricidia sepium, (Jacq.) Kunth ex Walp } \\
\text { Erythrina fusca, (Lour.) } \\
\text { Erythrina poeppigiana, (Walp.) O.F. Cook }\end{array}$ & $\begin{array}{l}\text { Madero negro } \\
\text { Poró } \\
\text { Poró }\end{array}$ \\
\hline Fabaceae Papilionoidea & Lonchocarpus sp. (Kunth) & $\begin{array}{l}\text { Chaperno-Corteza de venado-Pava-Pavilla- } \\
\text { Sietecueros }\end{array}$ \\
\hline Lauraceae & $\begin{array}{l}\text { Cinnamomum triplinerve, (Ruiz \& Pav.) } \\
\text { Kosterm. }\end{array}$ & Pedo de caballero \\
\hline Malvaceae & Guazuma ulmifolia, (Lam.) & $\begin{array}{l}\text { Guácimo-Capulín-Guácimo-Guácimo } \\
\text { ardilla-Guácimo blanco }\end{array}$ \\
\hline Malvaceae & $\begin{array}{l}\text { Pachira quinata, Alverson, William (Bil) } \\
\text { Surprison }\end{array}$ & Pochote \\
\hline Meliaceae & Cedrela odorata, $L$. & Cedro \\
\hline Moraceae & $\begin{array}{l}\text { Ficus sp. L. } \\
\text { Ficus benjamina } L .\end{array}$ & $\begin{array}{l}\text { Higuerón } \\
\text { Laurel de la India }\end{array}$ \\
\hline Muntingiaceae & Muntingia calabura $(L)$. & Capulín \\
\hline Myrtaceae & Psidium guajava, $(L)$. & Guayaba \\
\hline Primulaceae & Ardisia revoluta (Schltdl.) & Tucuico \\
\hline Rubiaceae & Randia karstenii & Escampa gallina \\
\hline Rutaceae & Zanthoxylum sp. (L.) & Lagartillo \\
\hline Solanaceae & $\begin{array}{l}\text { Cestrum sp. }(L) \\
\text { Solanum umbellatum (Mill). }\end{array}$ & Dama de noche, galán de noche. \\
\hline Urticaceae & Cecropia obtusifolia, (Bertol.) & Guarumo \\
\hline Urticaceae & $\begin{array}{l}\text { Urera caracasana, (Jacq.) Gaudich. ex } \\
\text { Griseb. }\end{array}$ & Ortiga \\
\hline
\end{tabular}

Se calcularon los parámetros descriptores de la estructura vegetal sobre abundancia, la frecuencia y la dominancia presente. Enterolobium cyclocarpum, se encuentra representada en los tres índices poblacionales mientras que la Bursera simaruba está entre las especies más abundantes y frecuentes. Guazuma ulmifolia se muestra como abundante y dominante; hacen la diferencia Tabebuia rosea dentro de las frecuentes y Ficus como más dominante (Cuadro 5). 


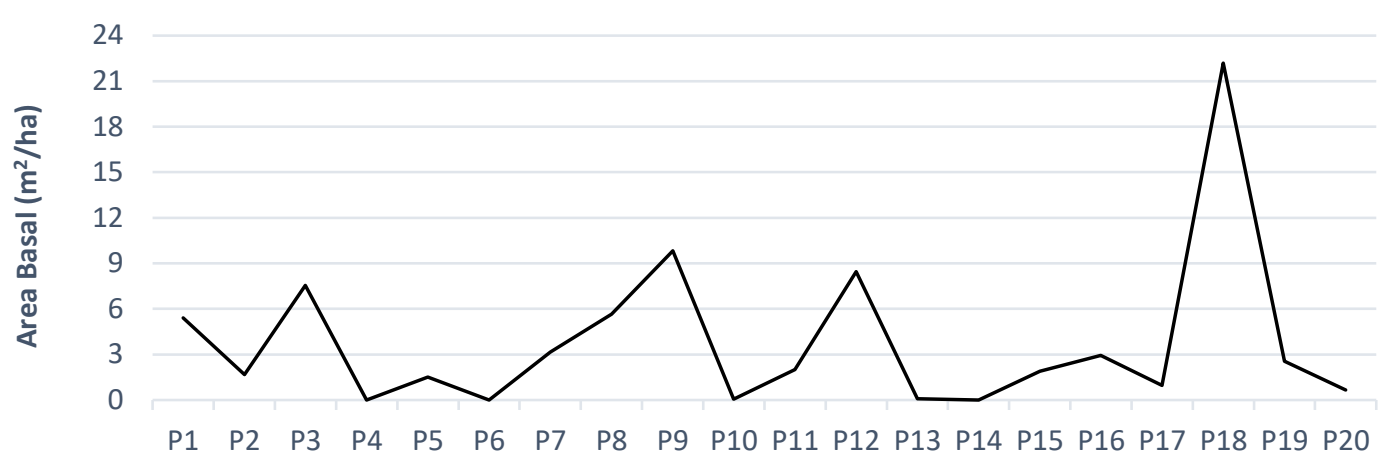

Figura 4. Área basal $\left(\mathrm{Ab} / \mathrm{m}^{2}\right)$ en parcelas de muestreo en la finca Los Llanos, La Garita, Alajuela.

Cuadro 2. Familias y especies identificadas en muestreo en parcelas la finca Los Llanos, 2016-2017

\begin{tabular}{|c|c|c|}
\hline Familia & Especie & Individuos \\
\hline Anacardiaceae & $\begin{array}{l}\text { Anacardium } \\
\text { excelsum }\end{array}$ & 2 \\
\hline Asteraceae & Vernonia patens & 2 \\
\hline Bignoniaceae & Tabebuia rosea & 7 \\
\hline Burseraceae & $\begin{array}{l}\text { Bursera } \\
\text { simaruba }\end{array}$ & 12 \\
\hline Euphorbiaceae & $\begin{array}{l}\text { Sapium } \\
\text { glandulosum }\end{array}$ & 3 \\
\hline Fabaceae & $\begin{array}{l}\text { Erythrina } \\
\text { berteroana }\end{array}$ & 3 \\
\hline Fabaceae Mimosoidea & $\begin{array}{l}\text { Enterolobium } \\
\text { cyclocarpum } \\
\text { Samanea saman }\end{array}$ & 17 \\
\hline $\begin{array}{l}\text { Fabaceae } \\
\text { Papilionoidea }\end{array}$ & Andira inermis & 7 \\
\hline Malvaceae & $\begin{array}{l}\text { Guazuma } \\
\text { ulmifolia }\end{array}$ & 8 \\
\hline Melastomastaceae & Miconia $s p$ & 1 \\
\hline Meliaceae & Cedrela odorata & 4 \\
\hline Moraceae & $\begin{array}{l}\text { Maclura } \\
\text { tinctorial } \\
\text { Ficus sp }\end{array}$ & 5 \\
\hline Myrsinaceae & $\begin{array}{l}\text { Ardisia } \\
\text { compressa }\end{array}$ & 1 \\
\hline Myrtaceae & Psidium guajava & 1 \\
\hline Rutaceae & Citrus limon & 1 \\
\hline Urticaceae & Cecropia sp & 2 \\
\hline
\end{tabular}

En cuanto al índice de valor de importancia (IVI) en la finca Los Llanos, se determinaron las 10 especies de mayor dominancia y el grado de heterogeneidad del ecosistema. Las especies de mayor importancia para esta zona son Enterolobium cyclocarpum, Bursera simaruba y Guazuma ulmifolia (Cuadro 5).

Cuadro 3. Especies clasificadas según gremios ecológicos, Los Llanos, 2016-2017

\begin{tabular}{lcl}
\hline \multicolumn{1}{c}{ Especies } & Cantidad & Gremios Ecológicos \\
\hline Cecropia peltata & 2 & Heliófita efímera \\
Bursera simaruba & 12 & Heliófita durable \\
Andira inermis & 7 & Heliófita durable \\
Ardisia compressa & 1 & Heliófita durable \\
Cedrela odorata & 4 & Heliófita durable \\
Enterolobium & & Heliófita durable \\
cyclocarpum & 16 & Heliófita durable \\
Sapium glandulosum & 3 & Heliófita durable \\
Tabebuia rosea & 7 & Heliófita durable \\
Guazuma ulmifolia & 8 & Heliófita durable \\
Samanea saman & 1 & Indeterminado \\
Citrus limon & 1 & Indeterminado \\
Psidium guajava & 1 & Indeterminado \\
Anacardium excelsum & 2 & Indeterminado \\
Erythrina berteroana & 3 & Indeterminado \\
Ficus sp & 2 & Indeterminado \\
Maclura tinctoria & 3 & Indeterminado \\
Miconia sp & 1 & Indeterminado \\
Vernonanthura patens & 2 &
\end{tabular}

En cuanto a las familias se identificaron las 10 principales según el índice de importancia Familia (IVF), las de mayor representación son la Fabaceae Mimosoidea, Moraceae y Malvaceae (Cuadro 6). 
Cuadro 4. Rangos de distribución por clase diamétrica de las especies muestreadas, Los Llanos, 2016-2017.

\begin{tabular}{cc}
\hline $\begin{array}{c}\text { Clase diamétrica } \\
(\mathbf{c m})\end{array}$ & $\begin{array}{c}\text { Abundancia } \\
\text { (arb/ha) }\end{array}$ \\
\hline $10-14.99$ & 3 \\
$15-19.99$ & 8 \\
$20-24.99$ & 4 \\
$25-29.99$ & 4 \\
$30-34.99$ & 7 \\
$35-39.99$ & 2 \\
$40-44.99$ & 1 \\
$45-49.99$ & 5 \\
$50-54.99$ & 2 \\
$55-59.99$ & 3 \\
$60-64.99$ & 1 \\
$65-69.99$ & 0 \\
$70-74.99$ & 1 \\
$75-79.99$ & 1 \\
& $80<$ \\
\hline
\end{tabular}

Finalmente, dentro de la caracterización del sitio de estudio se investigó sobre las condiciones edáficas y recurso hídrico. Los suelos observados corresponden al orden de vertisoles, se caracterizan por presentar arcillas expandibles con alto contenido en montmorillonita, que se contraen en época seca y se expanden en presencia de humedad. Son generalmente de color negros, forman profundas grietas en la estación seca y se vuelven muy duros. Mientras tanto, en la estación lluviosa se caracterizan por presentar una mayor plasticidad según el contenido de humedad (Figura 7).

Continuando con la caracterización del tipo de suelo presente en los Llanos, según el Dr. Wagner Peña (2016, com. pers.), indicó que el suelo presente en la finca Los Llanos es considerado dentro de los más fértiles en los trópicos estacionalmente secos. Se trata de un edafotaxon rico en bases, prevaleciendo calcio y magnesio en el complejo de intercambio catiónico, cuya

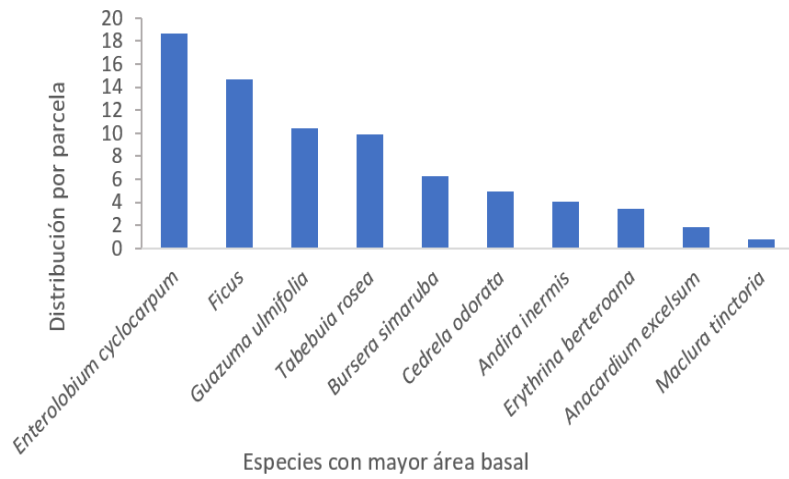

Figura 5. Las 10 especies con mayor área basal presente en el área de estudio, Los Llanos, La Garita, Alajuela.

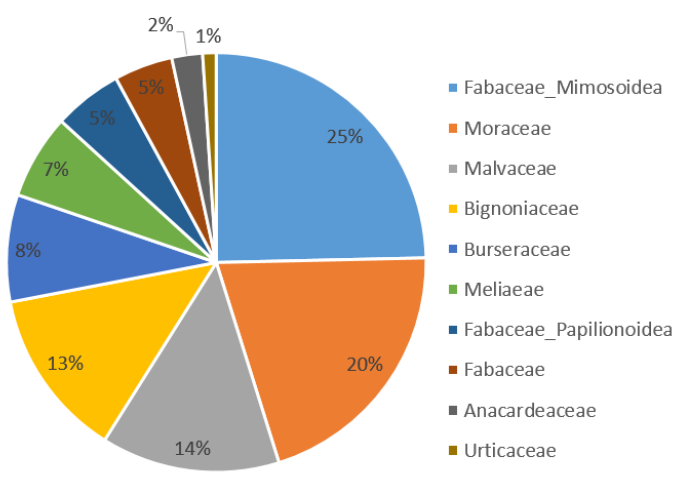

Figura 6. Familias en la finca Los Llanos, La Garita, Alajuela

solución oscila entre pH 6 y 7. Entre las propiedades físicas de este tipo de suelo se destaca la densidad de partículas superior a $1 \mathrm{~g} / \mathrm{cm}^{3}$, la baja conductividad hidráulica y la alta acumulación del carbono, genera condiciones que minimizan la velocidad de mineralización de los elementos esenciales. Es decir, las propiedades físico-mecánicas pueden provocar una acumulación de material entre las arcillas, por ejemplo, de materia orgánica, por lo que hay menor disponibilidad de nutrientes a pesar de la alta capacidad de intercambio que presentan estas partículas minerales, siendo una estrategia de regeneración ecológica el disponer de especies vegetales que favorezcan la mineralización y disponibilidad de nutrimentos.

En cuanto a la caracterización del tipo del recurso hídrico: Los ecosistemas acuáticos se clasificaron en dos condiciones generales del desplazamiento de aguas, los ecosistemas lóticos que contemplan las zonas riparias de ríos y áreas de nacientes, arroyos. 
Cuadro 5. Composición en cuanto abundancia, frecuencia, dominancia e índice de valor de importancia de las especies identificadas en Los Llanos, La Garita, Alajuela, 2016 -2017.

\begin{tabular}{lcccc}
\hline \multicolumn{1}{c}{ ESPECIES } & $\begin{array}{c}\text { Abundancia } \\
\text { relativa }\end{array}$ & $\begin{array}{c}\text { Frecuencia } \\
\text { relativa }\end{array}$ & $\begin{array}{c}\text { Dominancia } \\
\text { Relativa }\end{array}$ & $\begin{array}{c}\text { IVI a } \\
100 \%\end{array}$ \\
\hline Enterolobium cyclocarpum & 21,053 & 15,686 & 24,309 & 0,203 \\
Bursera simaruba & 15,789 & 13,725 & 8,158 & 0,126 \\
Guazuma ulmifolia & 10,526 & 9,804 & 13,596 & 0,113 \\
Tabebuia rosea & 9,211 & 11,765 & 12,884 & 0,113 \\
Ficus sp. & 2,632 & 3,922 & 19,205 & 0,086 \\
Andira inermis & 9,211 & 5,882 & 5,235 & 0,068 \\
Cedrela odorata & 5,263 & 5,882 & 6,459 & 0,059 \\
Erythrina berteroana & 3,947 & 3,922 & 4,463 & 0,041 \\
Sapium glandulosum & 3,947 & 5,882 & 0,226 & 0,034 \\
Maclura tinctoria & 3,947 & 3,922 & 1,061 & 0,030 \\
\hline
\end{tabular}

Cuadro 6. Índice de Valor de Importancia (IVF) para las especies registradas en la Finca en Los Llanos, La Garita, Alajuela, 2016 -2017.

\begin{tabular}{lcccc}
\hline FAMILIAS & $\begin{array}{c}\text { Abundancia } \\
\text { relativa }\end{array}$ & $\begin{array}{c}\text { Dominancia } \\
\text { Relativa }\end{array}$ & $\begin{array}{c}\text { DivF } \\
\text { relativa }\end{array}$ & $\begin{array}{c}\text { IVF } \\
100 \%\end{array}$ \\
\hline Fabaceae Mimosoidea & 22,368 & 24,350 & 11,111 & 0,193 \\
Moraceae & 6,579 & 20,267 & 11,111 & 0,127 \\
Malvaceae & 10,526 & 13,596 & 5,556 & 0,099 \\
Burseraceae & 15,789 & 8,158 & 5,556 & 0,098 \\
Bignoniaceae & 9,211 & 12,884 & 5,556 & 0,092 \\
Fabaceae Papilionoidea & 9,211 & 5,235 & 5,556 & 0,067 \\
Meliaeae & 5,263 & 6,459 & 5,556 & 0,058 \\
Fabaceae & 3,947 & 4,463 & 5,556 & 0,047 \\
Anacardeaceae & 2,632 & 2,343 & 5,556 & 0,035 \\
Euphorbiaceae & 3,947 & 0,226 & 5,556 & 0,032 \\
\hline
\end{tabular}
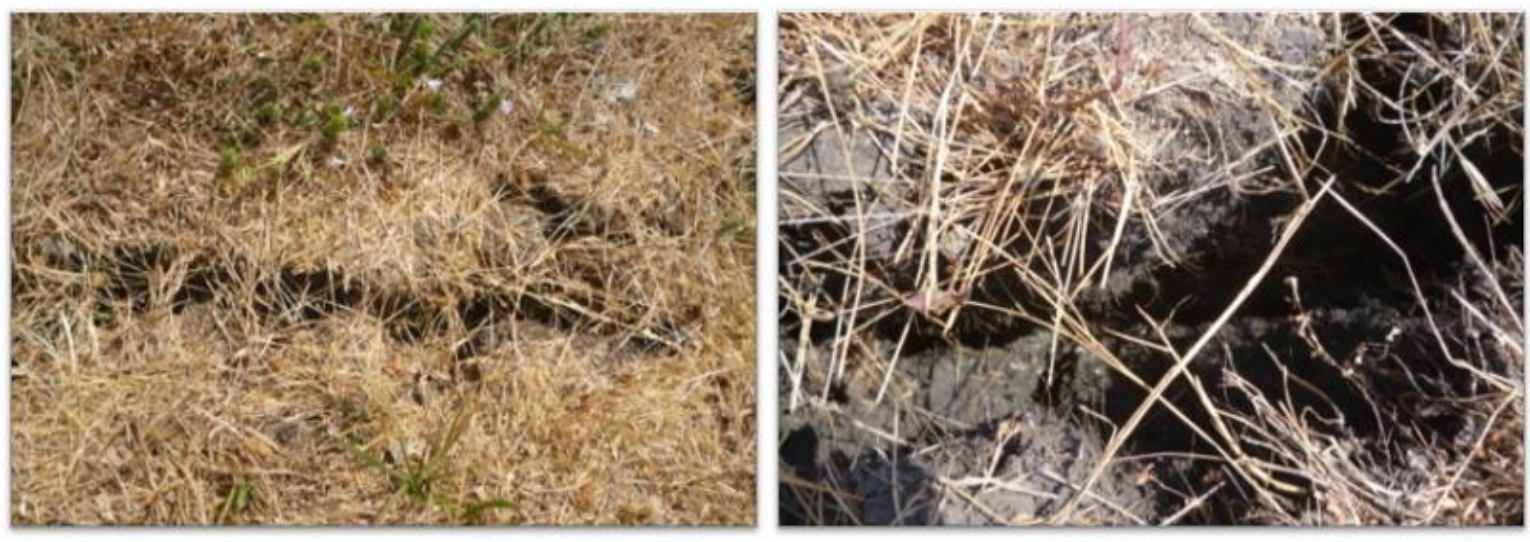

Fig. 7. Fotografías del tipo de suelo en época seca de la Finca Los Llanos, 2016-2017 

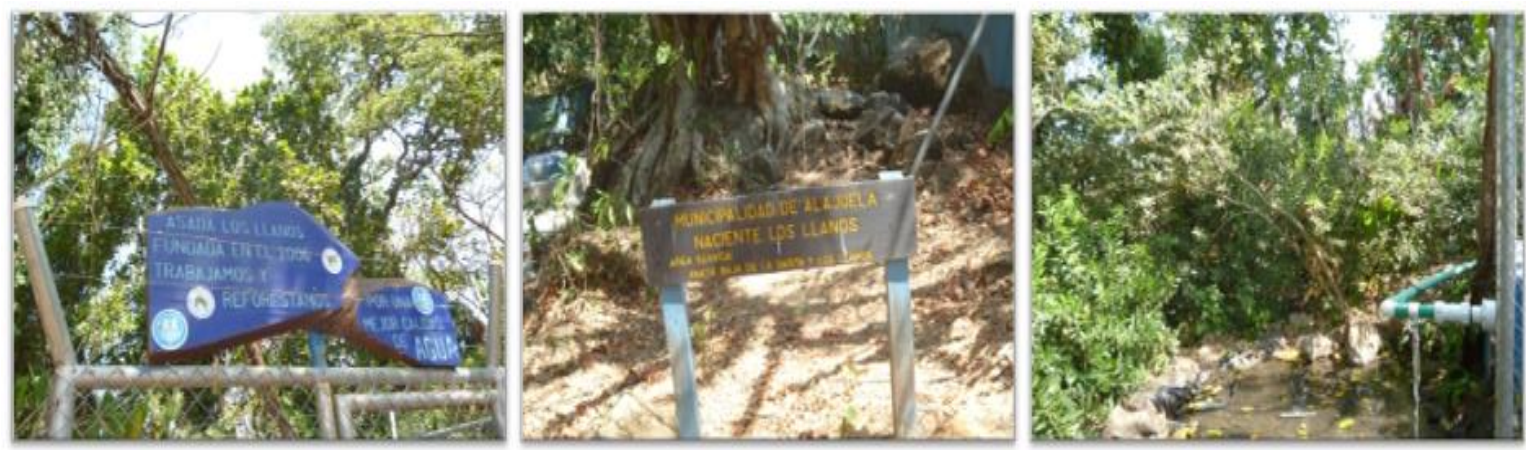

Figura 8. Excedente del tanque de captación de agua que se encuentra dentro de la finca y la naciente que esta adyacente a la propiedad, Alajuela, 2016 -2017.
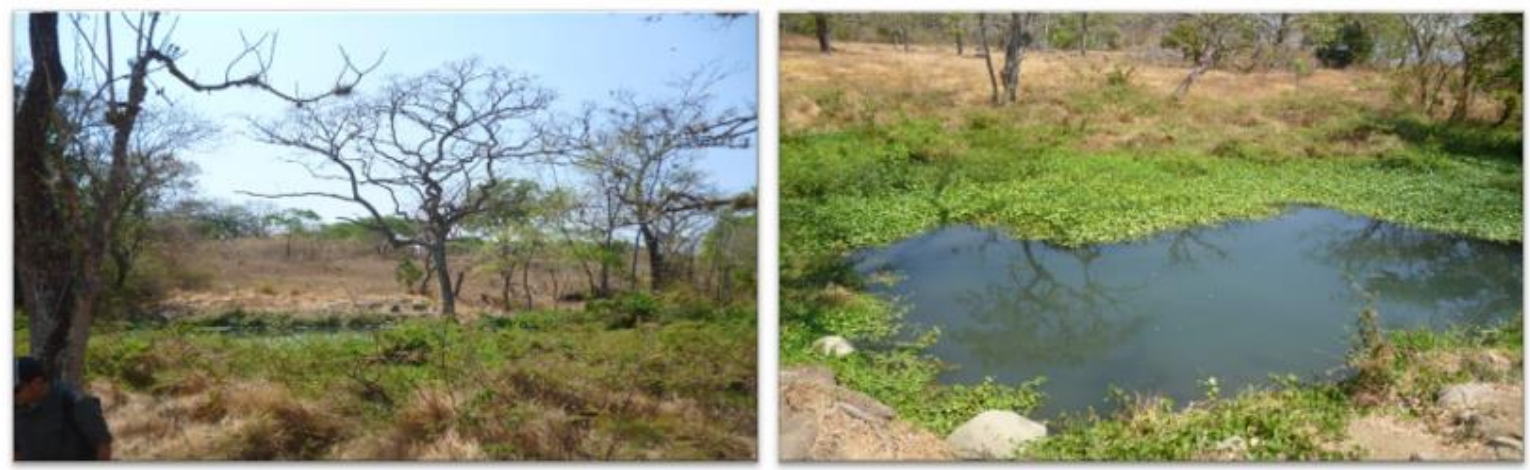

Figura 9. Foto de laguna, en época seca, marzo 2016 - 2017.

Y los ecosistemas lénticos, que se encuentran en los humedales, lagos y lagunas en este proyecto se cuentan con los dos tipos de condiciones generales de desplazamiento del agua (Figuras 8 y 9).

\section{Discusión}

El Bosque Húmedo Premontano (BHP) constituye uno de los ecosistemas más reducidos y fragmentados en Costa Rica y, a pesar de esto, los estudios que proporcionan información descriptiva apropiada para mejorar el conocimiento de este ecosistema son escasos (Cascante y Estrada, 2001). Con la presente investigación se contribuyó a la caracterización florística de una finca representativa de la zona de vida bosque húmedo premontano transición a basal. Este tipo de investigación es una herramienta necesaria para la implementación de medidas adecuadas para el manejo de sitios degradados y fragmentados.

La finca Los Llanos en su mayoría está compuesto por especies herbáceas y arbusto, sin embargo, estas protegen el suelo contra los agentes erosivos y contribuyen a sus actividades biológicas, que son responsables de la descomposición de la materia orgánica y la fijación del nitrógeno en el suelo (FAO, 2015).

Asimismo, se identificaron cultivos mixtos, estos también protegen los suelos contra los agentes erosivos (por ejemplo, agua y viento), mejoran la estructura de los suelos al <<echar〉> raíces y enriquecen los nutrientes del suelo al proporcionar la materia orgánica y establecer una relación simbiótica con las bacterias del suelo (FAO, 2015).

En cuanto a la composición florística, la riqueza de especies de las familias Fabaceae, Burceracea y Malvaceae concuerda con la tendencia reportada para los bosques neotropicales (Pennington, Lewis y Ratter, 2006). La flora leñosa registrada en la finca Los Llanos son especies típicas del bosque húmedo premontano transición a basal.

Se identificó una baja riqueza de especies y familia en Los Llanos, que según Fournier et al. (1985) para el Valle Central de Costa Rica debería de existir aproximadamente entre 44 y 78 especies por hectárea, sin embargo, para la finca en estudio se contabilizó 21 familias y 39 especies, esta baja riqueza se explica por el uso de suelo al que fue sometida en cuanto a la deforestación, a la agricultura y a la ganadería. 
Los suelos en la finca Los Llanos, son del orden de los vertisoles, son muy arcillosos, las especies establecidas en estos suelos deben presentar características como, ser de fácil adaptación al tipo de suelo, crecer en sitios perturbados, con cierto grado de aridez (Sánchez, Harvey, Grijalva, Medina, Vílchez, y Hernández, 2005). Como es el caso de las especies Enterolobium cyclocarpum, Bursera simaruba y Guazuma ulmifolia, identificadas en esta investigación como las más abundantes y dominantes, estas son especies recuperadores de terrenos degradados, son comunes en áreas húmedas y secas, proporcionan sombra y refugio a fauna, especies de rápido crecimiento e importantes para procesos de restauración ecológica (CONABIO 2018). Asimismo, Stokes (2001), menciona que estas especies se encuentran por lo general en fincas agroforestales debido a que proporcionan follaje y frutos para alimentar el ganado y proporcionar medicinas y alimentos a los propios ganaderos y a la fauna silvestre, lo cual explica la presencia de estas especies en Los Llanos.

Ahora bien, todas las especies identificadas se caracterizan por adaptarse fácilmente en áreas secas y húmedas, son de sitios abiertos, pastizales, márgenes de ríos y arroyos, sitios desmontados; crece en parcelas de cultivos, orilla de caminos, su crecimiento varía según sus condiciones ecológicas, estas especies se desarrolla de forma exitosa en tipos de suelo arenoso-arcilloso; características que cumplen en Los Llanos (CATIE, 1997).

La estructura vertical de la cobertura forestal de Los Llanos muestra poca presencia de sombra, Lamprecht (1990), en esta investigación las heliófitas durables fueron las más representativas, las cuales se establecen en etapas sucesionales tempranas por su requerimiento de luz. Actualmente existe un gran interés en ellas, por razones económicas y a que tienen altas tasas de crecimiento, este grupo incluye especies de alto valor comercial (SIREFOR, 2018).

El índice de ocupación espacial del área basal (parcelas, especies y familias), determinó el grado de ocupación mayor de cuatro especies y dos familias. En cuanto a las parcelas, la P18 sobresalió por contener especies con una alta distribución diamétrica esto describe el espacio de crecimiento de las especies. Donoso (1981), menciona que este crecimiento va a depender de las características bióticas y abióticas del lugar y que existe un conjunto de factores que inciden en el crecimiento y se refleja en la densidad.

Con relación a las condiciones edáficas, los autores Bergoeing y Protti, (1997), mencionan que el material parental de estos suelos, sobresalen las andesitas y rocas básicas ignimbríticas que proporcionan características de basicidad en los sustratos. El origen de estas rocas está asociado a la actividad del Volcán Poás, cuyo material fue depositado de forma extrusiva conocida como fila rocosa de Turrucares. Son rocas cuyas litologías corresponden al cuaternario, de una cronología del pleistoceno medio en un área volcánica determinada como meseta, se compone fundamentalmente de basaltos, que conforman la base volcánica que construyó la Cordillera Central a inicio del Cuaternario, compuestos por ignimbritas, tobas y lahares.

Se recomienda implementar un proyecto de restauración ecológica tomando en cuenta todos los resultados obtenidos y la caracterización en este estudio, que será de gran ayuda en el proceso inicial.

Es importante contemplar que la finca Los Llanos se encuentra parcialmente inmersa dentro del área de protección de dos nacientes, por lo que es necesario restaurar lo antes posible estos espacios, no obstante, Costa Rica cuenta con un amplio cuerpo normativo, en materia ambiental, que abarca desde la última Constitución Política de 1949, hasta otras leyes y decretos de importancia para la conservación, protección y restauración del paisaje natural como por ejemplo la Ley de Aguas N²76 de 1942 en sus artículos 31-32 y la Ley Forestal 7575. (Asamblea Legislativa).

\section{Agradecimiento}

Agradezco a Oscar Chacón Chavarría por sus aportes y comentarios y a María Auxiliadora Zúñiga por la ayuda en la identificación de especies forestales encontradas en la Finca Los Llanos. Esta investigación formó parte de la tesis de Maestría en Manejo de Recursos Naturales de la Universidad Estatal de la autora

\section{Referencias}

Asamblea Legislativa de la República de Costa Rica, 1994. Ley No.7412. Reforma constitucional. Constitución política de Costa Rica.

Asamblea Legislativa de la República de Costa Rica, 1996. Ley No 7575. Ley Forestal.

Bawa y McDade, 1994. The plant community: composition, dynamics, and life-history processes - Commentary, p.68. In L. McDade, K.S. Bawa, H. A. Hespenheide y G. S. Hartshorn (eds.). La Selva: ecology and natural history of a neotropical rain forest. The University of Chicago, Chicago, Illinois.

Bergoeing y Protti, 1997. Atlas Geomorfológico de Costa Rica, escala 1;350 000, ICE, UCR. 
Bonilla, 2018. Variación en composición y estructura de la vegetación leñosa de un bosque húmedo premontano transición seca, debido a la actividad agrícola y ganadera. Revista Cuadernos de investigación. Vol. 12. N 2.

Cascante y Estrada, 2001. Composición florística y estructural de un bosque húmedo premontano en el Valle Central de Costa Rica, Rev. Biología tropical, 49 (1): 213-225, 2001.

Cascante y Estrada, 2012. Diversidad y composición del fragmento más importante de bosque premontano del Valle Central de Costa Rica. Rev. BRENESIA 77:57-70, San José, Costa Rica.

CATIE, 1997. Nota técnica no52, base de datos. Recuperado de: http://orton.catie.ac.cr/repdoc/A0008s/A0008 s52.pdf

CONABIO 2018. Comisión Nacional para el Conocimiento y Uso de la Biodiversidad. Recuperado de:

http://www.conabio.gob.mx/conocimiento/inf o_especies/arboles/doctos/41-legum16m.pdf

Donoso, 1981. Ecología Forestal. El bosque y su medio ambiente. Editorial Universitaria., Universidad Austral de Chile. Recuperado de: http://amazoniaforestal.blogspot.com/2011/10 /medicion-de-diametro.html

FAO, 2015. Los suelos constituyen la base de la alimentación. Recuperado de: http://www.fao.org/fileadmin/user_upload/so ils-

2015/docs/Fact_sheets/Es_IYS_Veg_Print.pd f

Fournier, Flores y Rivera, 1985. Flora arborescente del Valle Central de Costa Rica. San José, Costa Rica. Jiménez y Tanzi, 149 p.

Harvey, Haber, 1999. Remanent trees and the conservation of biodiversity in Costa Rican pastures. Agrof. Systems. 44: 37-68.

IMAS, 2013. Instituto Mixto de Ayuda Social. Sesión ordinaria de consejo directivo celebrada el lunes 17 de junio de 2013 acta No. 041-062013. Recuperado de: http://www.imas.go.cr/acerca_imas/transpare ncia/actascd/cd-2013/ACTA-041-062013_17-junio.pdf

Lamprecht, 1990. Silvicultura en los trópicos: los ecosistemas forestales en los bosques tropicales y sus especies arbóreas; posibilidades y métodos para un aprovechamiento sostenible; trad. Antonio Carrillo; Deutsche; GTZ, Gmdh.

Moreno, C. E. 2001. Métodos para medir la biodiversidad. M\&T-Manuales y Tesis SEA, vol. 1. Zaragoza, 84 pp

Mori, S. A., BooM, B. M., de Carvalho, A. M., \& dos Santos, T. S. (1983). Southern Bahian moist forests. The Botanical Review, 49(2), 155-232.

Mostacedo, B. \& Fredericksen, T. (2000). Manual de Métodos Básicos de Muestreo y Análisis en ecología vegetal. Santa Cruz de la Sierra, Bolivia: BOLFOR.

Pennington, Lewis y Ratter, 2006. Neotropical savannas and seasonally dry forests: plant diversity, biogeography, and conservation. Economic Botany, 61(3), 311-312. http://doi. org/10.1663/0013-0001(2007)61[311b: NSASDF]2.0.CO;2

Sánchez y Azofeifa, 2001. Medida y estructura interna del sentimiento de comunidad: un estudio empírico. Revista de Psicología Social, 16 (2), 157-175

Sánchez, Harvey, Grijalva, Medina, Vílchez y Hernández, (2005). Diversidad, composición y estructura de la vegetación en un agropaisaje ganadero en Matiguás, Nicaragua. Revista de biologia tropical, 53(3-4), 387-414.

SIREFOR, 2018. Sistema de Información de los Recursos Forestales de Costa Rica (SIREFOR), Costa Rica. Recuperado de: http://www.sirefor.go.cr/?page_id=724

Stokes, 2001. Farmers' knowledge about the management and use of trees on livestock farms in the Cañas area of Costa Rica. Thesis Mag. Sc. Bangor, UK, University of Wales. $78 \mathrm{p}$.

Villarreal, H., Álvarez, M., Córdoba, S., Escobar, F., Fagua, G., Gast, F., ... \& Umaña, A. M. (2006). Métodos para el análisis de datos: una aplicación para resultados provenientes de caracterizaciones de biodiversidad. Manual de Métodos Para el Desarrollo de Inventarios de Biodiversidad. Instituto de Investigación de Recursos Biológicos Alexander von Humboldt, Bogotá, Colombia, 185226. 